\title{
Assembly conditions for mechanical systems with gear elements
}

\author{
Dmitry Kurasov* \\ Kurgan State University, Kurgan region, Kurgan, Russia, 640020
}

\begin{abstract}
The article describes the general approach to compiling the assembly conditions of mechanical systems with toothed elements, in particular gear eccentric system (GES), and any toothed ones using the «toothed contours» method. Design dependencies constituting the engineering calculation algorithm are necessary for design and its technical use.
\end{abstract}

\section{Introduction}

Mechanical systems with gear elements, planetary gears and gear eccentric system (GES) built on their basis are widespread in mechanical engineering. Each separately considered design requires the fulfillment of certain geometric conditions, in particular, assembly conditions. The assembly condition is to ensure the correct engagement of gear wheels that form a closed loop, with a predetermined relative location of their centers. Currently, quantitative dependencies characterizing the condition for assembling various specific planetary mechanisms are usually given in the literature in the form of calculation formulas deduced specifically for these structures of mechanisms. However, the already known and newly synthesized schemes of gear mechanisms are very diverse and in order to carry out their geometric calculations, the engineer must have a general method of compiling equations describing the conditions of assembly. A general principle approach to compiling assembly conditions for mechanisms, each toothed link of which has one toothed rim, is given in the monograph by [1]. It consists in considering the contours composed of the «continuing» arcs of the initial circles of the interacting wheels, forming a kind of «gear chain». According to [1], the total length of these arcs (the distances between the poles of the respective engagements) must be a multiple of the number of separation steps of the engagement. In the particular case when the initial circles of all wheels coincide with the dividers, the application of this approach is the most obvious. However, this approach works in the general case where the initial circles do not coincide with the dividers. In this case, the integer must be the sum of the dividing steps $t$ in the loop (otherwise, the number of steps as abstract discretes). The very problem of the condition of assembly arises precisely in the presence of closed contours of engaging gears.

\footnotetext{
*Corresponding author: naukka@mail.ru
} 


\section{Gear eccentric system (GES)}

In mechanical engineering, mechanisms are widespread that convert rotational motion into reciprocating or oscillating. Most often they are used in combination with gearboxes. Such mechanisms can be obtained from mechanical systems with gear elements.

The design solution combining the functions of the above-mentioned transforming mechanisms and reduction gear provides for the use of the so-called GES [2] (Fig. 1). It contains driving shaft 1 , coaxially fixed on it gear 2, outer ring 3 with internal teeth, toothed rolling bodies (satellites) of different diameter 4 (which provides eccentricity eh), driven link 5 and articulated connecting rod 6 . Teeth of pinion rims 2, ring 3 and satellites 4 are made concentric to cylindrical running paths, diameters of which are equal or close to corresponding initial diameters of toothed rims.

GES in its structure is a planetary mechanism and the geometric conditions of the existence of GES are essentially similar to similar conditions for coaxial planetary mechanisms, but their mathematical expressions are completely different [3]:

- neighborhoods;

- correspondence of the number of wheel teeth to their location (actual assembly condition);

- correspondence of initial wheel diameters to their location (instead of coaxial condition for coaxial planetary mechanisms).

If the calculated expressions of the intrinsic condition of the GES are similar to the case of coaxial gears, then the other two geometric conditions of the GES will be completely different than for ordinary planetary gears.

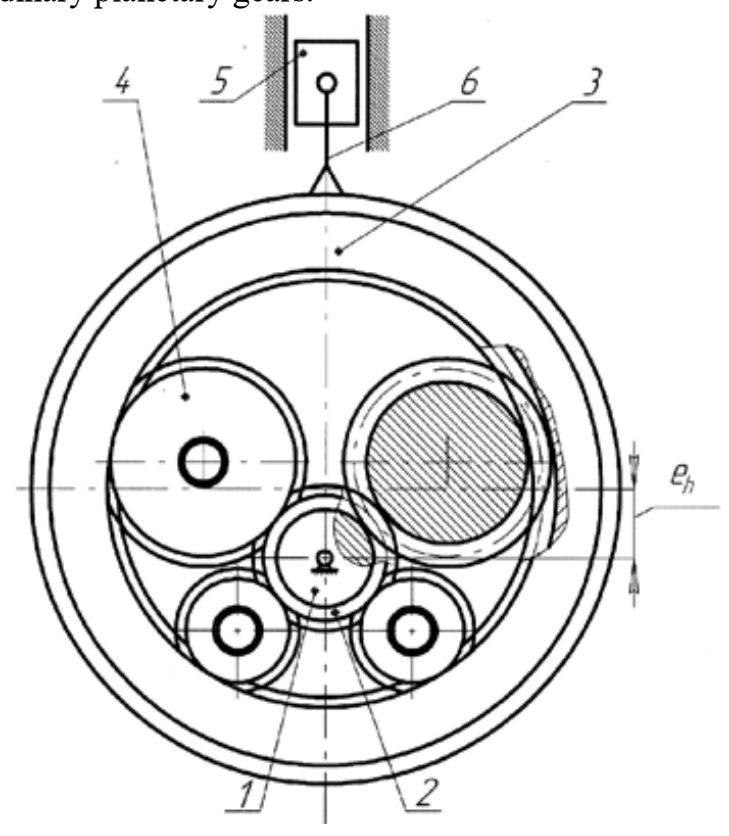

Fig. 1. Gear eccentric system (GES)

\section{Assembly condition}

For a conventional planetary transmission with one layer of satellites, with coaxial wheels, the equation corresponding to the assembly condition will be: 


$$
\frac{Z_{3}+Z_{1}}{k}=C
$$

where $\mathrm{k}$ is the number of satellites and $\mathrm{C}$ is integer value.

Using the method of "gear chains" [4], you can describe the assembly conditions for more complex mechanical systems.

Design dependencies of the GES assembly condition are written in the form of a system of equations, each of which corresponds to a certain pair of satellites (Fig. 2) located between the gear rims of the driving gear and the outer ring.

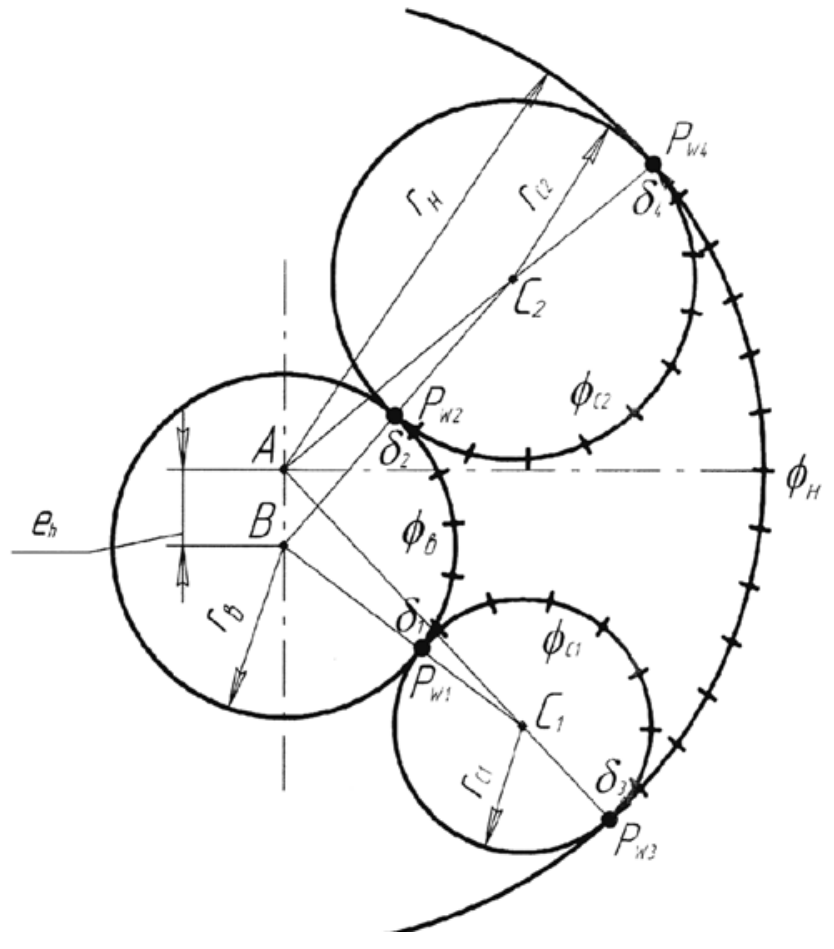

Fig. 2. Design diagram of SEP assembly condition

For a particular case, when all gear wheels are cut without displacement, and their dividing circles coincide with the initial circles of the length of the arcs $\cup \phi_{B}, \cup \phi_{H}, \cup \phi_{C 1}$ and $\cup \phi_{C 2}$ :

$$
\begin{aligned}
& \cup \phi_{B}=P \cdot z_{B}^{\prime}+\delta_{1}+\delta_{2}, \\
& \cup \phi_{H}=P \cdot z_{H}^{\prime}+\delta_{3}+\delta_{4}, \\
& \cup \phi_{C 1}=P \cdot z_{C 1}^{\prime}+\delta_{1}+\delta_{3}, \\
& \cup \phi_{C 2}=P \cdot z_{C 2}^{\prime}+\delta_{2}+\delta_{4},
\end{aligned}
$$

where is $P$ the pitch of the teeth along the pitch circle; $z_{B}^{\prime}, z_{H}^{\prime}, z_{C 1}^{\prime}, z_{C 2}^{\prime}$ is the number of full pitch of the teeth located on the arcs $\cup \phi_{B}, \cup \phi_{H}, \cup \phi_{C 1}$ and $\cup \phi_{C 2} ; \delta_{1}, \delta_{2}, \delta_{3}, \delta_{4}$, is the length of the arcs corresponding to the partial pitch.

After the transformations, we get the assembly condition: 


$$
\cup \phi_{B}+\cup \phi_{H}-\cup \phi_{C 1}-\cup \phi_{C 2}=P \cdot K
$$

where $K=z_{b}^{\prime}+z_{H}^{\prime}-z_{C 1}^{\prime}-z_{C 2}^{\prime}$ parameter is an integer.

The universal equation of the GES assembly condition for a pair of satellites will be:

$$
\phi_{B} \cdot z_{B}+\phi_{H} \cdot z_{H}-\phi_{C 1} \cdot z_{C 1}-\phi_{C 2} \cdot z_{C 2}-2 \cdot \pi \cdot K=0
$$

where $\phi_{\theta}, \phi_{H}, \phi_{C 1}, \phi_{C 2}$ is the central angles resting on the poles of the corresponding engagements, for each of the wheels, measured inside the contour formed by the poles $P_{w 1}, P_{w 2}$ and $P_{w 3}, P_{w 4} ; z_{b}, z_{H}, z_{C 1}, z_{C 2}$ is the full number of teeth of these wheels.

Equivalent assembly condition record form:

$$
\phi_{B} \cdot z_{B}+\phi_{H} \cdot z_{H}+\phi_{C 1}^{\prime} \cdot z_{C 1}+\phi_{C 2}^{\prime} \cdot z_{C 2}-2 \cdot \pi \cdot K^{\prime}=0,
$$

where $\phi_{C 1}^{\prime}, \phi_{C 2}^{\prime}$ are the central angles of the satellites resting on the engagement poles measured from the outside of the pole contour; $K^{\prime}$ is another integer (it corresponds to the number of links of the "chain" worn on satellites as asterisks).

The parameters of equations (7) and (8) are related by the relations: $\phi_{C 1}^{\prime}=2 \cdot \pi-\varphi_{C 1}$ $; \phi_{C 2}^{\prime}=2 \cdot \pi-\phi_{C 2} ; K^{\prime}=K+z_{C 1}+z_{C 2}$.

\section{Condition of conformity of initial diameters of wheels to their location}

The calculation scheme for this condition is shown in Fig. 3. On $r_{\omega C}, r_{\omega B}, r_{\omega H}$ it are the radius of the initial circles of the satellite, gear and outer ring, respectively; $e_{h}=\mathrm{AB}$ is the eccentricity of the gear relative to the outer ring; $\psi_{H}$ is an angle located between a line passing along the eccentricity line and a line passing from the point $\mathrm{A}$ of the center of the GES (that is the center of the circle of the radius $r_{\omega H}$ ) through the point $\mathrm{C}$ of the center of the satellite.

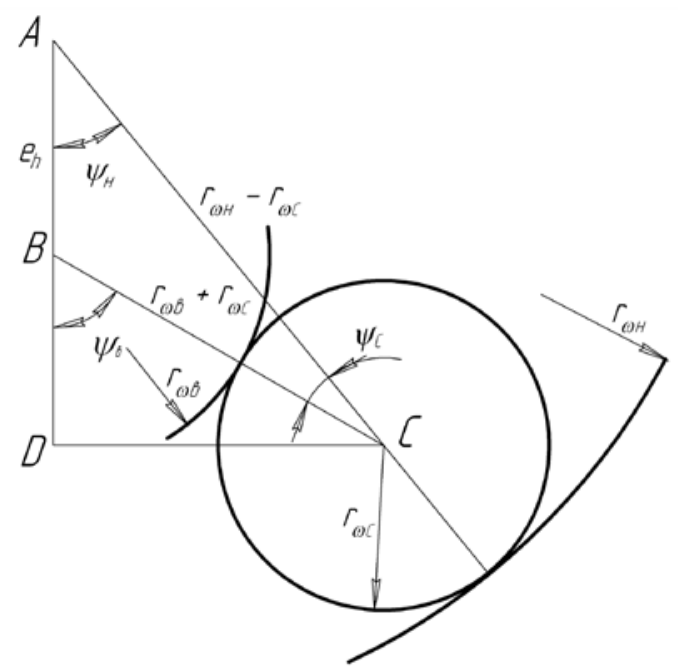

Fig. 3. Design diagram of condition of compliance of initial diameters of wheels with their arrangement 
The calculated equations linking the initial diameters $\psi_{C}, \psi_{B}, \psi_{H}$ of the wheels and the satellite can be obtained in various ways.

In calculations that do not require explicit expression of angles, it is more convenient to use simpler equations derived from the vector contour method [5]. For the ACB triangle, the closed vector loop equation:

$$
A B+A C-B C=0 .
$$

When projecting (9) onto the coordinate axes $\mathrm{X}$ and $\mathrm{Y}$, we obtain the following system of equations:

$$
\begin{gathered}
\left(z_{H} \cdot m_{\omega i}-z_{C} \cdot m_{\omega i}\right) \cdot \cos \psi_{H}+\left(z_{B} \cdot m_{\omega i}+z_{C} \cdot m_{\omega i}\right) \cdot \cos \psi_{B}=0, \\
2 \cdot e_{h}+\left(z_{H} \cdot m_{\omega i}-z_{C} \cdot m_{\omega i}\right) \cdot \sin \psi_{H}+\left(z_{B} \cdot m_{\omega i}+z_{C} \cdot m_{\omega i}\right) \cdot \sin \psi_{B}=0 .
\end{gathered}
$$

The features of GES are that their kinematic schemes with three or more satellites are statically undefined, and most of the geometric parameters of $\operatorname{GES}\left(z_{H}, z_{B}, z_{C 1}, z_{C 2}\right.$ and $K_{1}, K_{2}$ ) are integer. Under such conditions, the choice of parameters that allow monotonous variation in solving the system of equations connecting the geometric parameters of the GES is limited. In addition to the angles, these are eccentricity $e_{h}$ and initial modules $m_{\omega i}$, which can be selected independently for each engagement. However, if there are different initial modules in different engagements, then one wheel in different engagements will have different initial diameters. At the same time, it is impossible to ensure simultaneous equality of the initial diameters of the gear rims with the diameters of the treadmills for each wheel. As a result, some slippage will occur on the treadmills, which is undesirable. You can limit yourself to varying only the parameter $e_{h}$ only in the case of two satellites.

It is proposed to introduce an additional parameter $\Delta$ is radial tension in kinematic pairs, reduced to the radius of the outer ring $r_{\omega H}$, expressed in fractions of the module: $\Delta=\Delta r_{\omega H} / m_{\omega}$.

Thus, the actual initial radius of the outer ring:

$$
r_{\omega H}-\Delta r_{\omega H}=\frac{z_{H} \cdot m_{\omega}}{2}-\Delta \cdot m_{\omega}
$$

Taking this into account, the system of equations is converted to the form:

$$
\begin{gathered}
\left(z_{H}-2 \cdot \Delta-z_{C}\right) \cdot \cos \psi_{H}+\left(z_{B}+z_{C}\right) \cdot \cos \psi_{B}=0 ; \\
2 \cdot e+\left(z_{H}-2 \cdot \Delta-z_{C}\right) \cdot \sin \psi_{H}+\left(z_{B}+z_{C}\right) \cdot \sin \psi_{B}=0,
\end{gathered}
$$

where $e=e_{h} / m_{\omega}$ is the eccentricity expressed in parts of the module.

\section{Algorithm for geometric calculation of SEP according to assembly conditions}

1 stage. Determining the numbers of planet teeth $z_{C 1}, z_{C 2}$ and integer parameters: $K_{l}$, referring to two small satellites, and $K_{2}$, referring to small and large satellites.

We round the resulting values $z_{C 1}, z_{C 2}, K_{1}, K_{2}$ to integers. At the same time, it is advisable to consider several rounding options. 
Stage 2. Refinement of GES parameters for received values $z_{H}, z_{B}, z_{C 1}, z_{C 2}, K_{1}, K_{2}$.

Among the results of the solution, you need to select those that contain acceptable interference values $\Delta$. In the absence of such, the calculation for the changed initial data should be repeated.

Check of neighborhood condition is performed according to principles common with coaxial planetary gears (Fig.4). The calculated expression for this condition: $r_{a 1}+r_{a 2}<l_{1,2}$, where $l_{1}, l_{2}$ are the axial distances between the corresponding satellites; $r_{a 1}, r_{a 2}$ is the radius of the vertices of the teeth of the satellites.

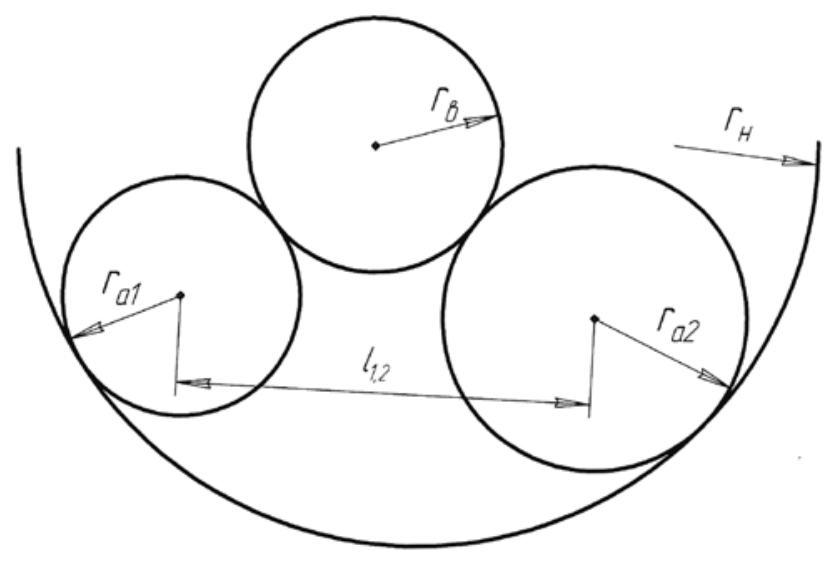

Fig. 4. Neighborhood condition

\section{Conclusions}

GES is a fairly simple mechanism based on the principle of a planetary mechanism, the use of which will make the drives of many machines more compact and make them cheaper. Unlike coaxial planetary gears, constraints for assembly conditions are written in the form of a system of equations, each of which corresponds to a certain pair of satellites. The assembly approach described above is suitable for most mechanical systems with gear elements and is an algorithm for engineering calculations necessary for design and its technical use.

\section{References}

1. Kudryavtsev V N 1966 Planetary gear (L.: Engineering) p 308

2. Volkov G Yu, Kurasov D A 2008 Cageless roller bearing, Pat. of the Russian Federation No. 2,331,000

3. Kudryavtsev V N 1977 Planetary gear: Reference book (L.: Engineering) p 536

4. Volkov G Yu, Ratmanov E V, Kurasov D A and Kolmakov S V 2010 Planetary transmission assembly conditions with two satellite layers 10 22-6

5. Volkov G Yu, Kurasov D A 2008 Modification of slide motion in a crank-slide mechanism by introducing an eccentric gear system Russian Engineering Research 5 403-6. 